\title{
Matrix metalloproteinases and soluble Fas/FasL system as novel regulators of apoptosis in children and young adults on chronic dialysis
}

\author{
Kinga Musiał • Danuta Zwolińska
}

Published online: 23 April 2011

(c) The Author(s) 2011. This article is published with open access at Springerlink.com

\begin{abstract}
The system of membrane receptor Fas and its ligand FasL compose one of the main pathways triggering apoptosis. However, the role of their soluble forms has not been clarified yet. Although sFasL can be converted from the membrane-bound form by matrix metalloproteinases (MMPs), there are no data on relations between sFas/ sFasL, MMPs and their tissue inhibitors (TIMPs) in patients on chronic dialysis-neither children nor adults. The aim of our study was to evaluate serum concentrations of sFas, sFasL, and their potential regulators (MMP-2, MMP-7, MMP-9, TIMP-1, TIMP-2), in children and young adults chronically dialyzed. Twenty-two children on automated peritoneal dialysis (APD), 19 patients on hemodialysis (HD) and 30 controls were examined. Serum concentrations of sFas, sFasL, MMPs and TIMPs were assessed by ELISA. Median values of sFas, sFasL, sFas/ sFasL ratio, MMP-2, MMP-7, MMP-9, TIMP-1 and TIMP-2 were significantly elevated in all dialyzed patients vs. controls, the highest values being observed in subjects on HD. A single HD session caused the decrease in values of all parameters to the levels below those seen in children on APD. Regression analysis revealed that MMP-7 and TIMP-1 were the best predictors of sFas and sFasL concentrations. Children and young adults on chronic dialysis are prone to sFas/sFasL system dysfunction, more pronounced in patients on hemodialysis. The correlations between $\mathrm{sFas} / \mathrm{sFasL}$ and examined enzymes suggest that MMPs and TIMPs take part in the regulation of cell death
\end{abstract}

K. Musiał · D. Zwolińska ( $\square)$

Department of Pediatric Nephrology, Wrocław Medical

University, M. Skłodowskiej - Curie 50/52,

50-369 Wrocław, Poland

e-mail: zwolin@nefped.am.wroc.pl in the pediatric population on chronic dialysis, triggering both anti- (sFas) and pro-apoptotic (sFasL) mechanisms.

Keywords Hemodialysis - Peritoneal dialysis . MMP-7 · TIMP-1

\section{Introduction}

Patients with chronic kidney disease (CKD) are prone to enhanced apoptosis, additionally accelerated by the dialysis procedure and the type of membrane that has been used [1, 2]. The system of membrane receptor Fas and its ligand FasL compose one of the main pathways leading to programmed cell death. Fas, expressed on a wide variety of cells, binds to its ligand, restricted mainly to macrophages, $\mathrm{T}$ lymphocytes and endothelial cells, thus triggering the apoptotic pathway [3].

Recent investigation has correlated these events with vascular damage and subsequent progression of atherosclerosis, being another hallmark of CKD [4]. Indeed, vascular smooth muscle cells (VSMCs) are resistant to apoptosis in atherosclerotic lesions [5] and sensitive to it in normal vessels [6]. Therefore, it has been suggested that FasL-induced apoptosis in normal vessel wall acts in a protective way by decreasing neointimal hyperplasia and thickening [7], whereas in atherosclerosis this mechanism seems ineffective, paradoxically increasing cell migration and inflammation [5].

The soluble forms of death receptor and its ligand, sFas and $\mathrm{sFasL}$, have recently been discovered, but their role in control of apoptosis has not been clarified yet. sFasL is proteolytically shed from its membrane-bound form by enzymes-matrix metalloproteinases (MMPs) [8]. The strongest evidence for such activity has been shown in 
in vitro experiments concerning matrilysin (MMP-7) [9]. The applicability of this regulatory pathway in clinical practice has focused on overcoming drug resistance to chemotherapy $[10,11]$ or controlling mesangial inflammation [12], but it has not been considered in the light of chronic kidney disease so far. The pro-apoptotic activity of sFasL is decreased in comparison to that of the membrane FasL [13]. Therefore, there is controversy whether such conversion from the membrane-bound to the circulating form may be regarded as an anti-apoptotic mechanism, controlled by matrix metalloproteinases, or is it still the pro-apoptotic feature in the sFasL characteristics that prevails [14]. Despite evidence for pro-apoptotic and antiinflammatory activity of FasL, taking part in protection against neointimal hyperplasia after vascular injury [7], the parallel profile has not been proven in the case of sFasL.

sFas acts in an anti-apoptotic way by blocking both FasL and sFasL in a 1:1 proportion. It has also been defined as a marker of inflammation and endothelial dysfunction in adults with coronary artery disease and chronic kidney disease $[15,16]$. Experimental data have shown the role of sFas in protecting the vessel wall integrity against injury [17]. However, the role of matrix metalloproteinases in sFas shedding is unknown and there are no data on relations between sFas/sFasL, MMPs, their tissue inhibitors (TIMPs) and inflammation/endothelial function in patients with end stage renal disease. The investigation comparing those parameters in the subjects treated with different dialysis modalities is lacking either.

Therefore, our aim was to assess the serum levels of $\mathrm{sFas}, \mathrm{sFasL}$ and the values of $\mathrm{sFas} / \mathrm{sFasL}$ ratio as a marker of overall apoptotic activity, in children and young adults on peritoneal dialysis and hemodialysis, searching for the differences between those modalities. We also investigated the correlations between sFas, sFasL, their potential regulators-matrix metalloproteinases (MMP-2, MMP-7 and MMP-9) and their tissue inhibitors (TIMP-1 and TIMP-2), as well as clinical significance of these findings, in the pediatric population on dialysis.

\section{Materials and methods}

Seventy-one patients enrolled in the study were divided into three groups. Data are shown in Table 1.

The first group consisted of 22 children on automated peritoneal dialysis (APD-Baxter, Home choice), 5 of them having residual renal function. The patients had 5-8 exchanges during the night and, if necessary, one or two during the day. Peritoneal fluids used in our patients had glucose concentrations varying from 1.36 to $2.27 \%$. The causative factors in CKD were: chronic pyelonephritis (seven cases), chronic glomerulonephritis (six), polycystic kidney disease (two), neurogenic bladder (three), hemolytic uremic syndrome (two) and unknown (two).

The second group included nineteen children and young adults hemodialyzed (HD) on polysulfone (PS) membranes, only three out of them with residual kidney function. HD sessions $(3.5-4 \mathrm{~h})$ were performed three times a week, using bicarbonate dialysate, the blood flow ranged from 150 to $200 \mathrm{ml} / \mathrm{min}$, dialysate flow did not exceed $500 \mathrm{ml} / \mathrm{min}$. The membrane area was between 1.0 and $1.6 \mathrm{~m}^{2}$, the dialyzers were not reused. The water, purified by reosmosis, was regularly checked for contamination. All patients were on stable anticoagulation regimen using lowmolecular-weight heparin. The causative factors in chronic renal failure were: chronic glomerulonephritis (seven cases), chronic pyelonephritis (seven), neurogenic bladder (two), polycystic kidney disease (one) and unknown (two).

Thirty age-matched children with primary nocturnal enuresis and normal kidney function, served as controls.

None of the patients showed clinical evidence of infection, had diabetes, malignancies or vasculitides, smoked, took antibiotics, statins, corticosteroids or immunosuppressive therapy. All APD children had their blood pressure values below the 90th percentile, adjusted for gender and age, according to the criteria of the fourth report on high blood pressure in children and adolescents [18], and did not require anti-hypertensives. The blood pressure in our HD patients was within normal values without medication (12 patients) or was well controlled with the use of ACE inhibitors only (3) or ACE inhibitors with calcium channel blockers (4).

Informed consent was obtained from the subjects and their parents, if necessary. The research project has been approved by the University ethics committee, in accordance with the Helsinki declaration.

Blood samples were drawn after an overnight fast from peripheral veins in APD patients and controls, in HD subjects-from the afferent line of the first-use dialyzer before starting an HD session. Samples were clotted for $30 \mathrm{~min}$, centrifuged at $4^{\circ} \mathrm{C}$ for $10 \mathrm{~min}$, and then serum was stored at $-20^{\circ} \mathrm{C}$ until assayed. Serum concentrations of sFas, sFasL, MMP-2 (gelatinase A), MMP-7 (matrilysin), MMP-9 (gelatinase B), TIMP-1 and TIMP-2 were evaluated by commercially available ELISA kits (R\&D Systems, Abingdon, UK). Standards and serum samples were transferred to 96 well microplates pre-coated with recombinant antibodies to human sFas, sFasL, MMP-2, MMP-7, MMP-9, TIMP-1 and TIMP-2. Each sample was tested in duplicate and the arithmetical mean was considered a final result. Measurements were performed according to the manufacturer's instructions, results were calculated by reference to standard curves. 
Table 1 The median values and interquartile ranges of basic demographical data and examined parameters in the patients on peritoneal dialysis (APD), on hemodialysis (HD) and in the control group

\begin{tabular}{|c|c|c|c|c|c|}
\hline \multirow[t]{2}{*}{ Parameter } & \multicolumn{5}{|c|}{ Median values (interquartile ranges) of analyzed parameters } \\
\hline & \multicolumn{2}{|c|}{ Control gr. $(n=30)$} & \multicolumn{2}{|c|}{$\mathrm{HD}(n=19)$} & $\operatorname{APD}(n=22)$ \\
\hline Age (years) & \multicolumn{2}{|c|}{$10.0(5.5-15.5)$} & \multicolumn{2}{|c|}{$13.5(10.5-17.0)$} & $10.0(4.0-15.5)$ \\
\hline Gender & \multicolumn{2}{|c|}{16 girls; 14 boys } & \multicolumn{2}{|c|}{10 girls; 9 boys } & 12 girls; 10 boys \\
\hline \multirow[t]{2}{*}{ Time of therapy } & \multirow[t]{2}{*}{-} & & \multicolumn{2}{|c|}{2.2 year $(1.0-2.7)$} & 2.0 year $(0.7-2.5)$ \\
\hline & & & & After HD & \\
\hline hsCRP (mg/l) & $0.62(0.28-1.34)$ & & & $0.91(0.21-7.69)$ & $0.59(0.29-1.40)$ \\
\hline MMP-2 (ng/ml) & $109.93^{\#}(90.47-116.98)$ & & 49.49) & $197.99(195.47-202.09)^{*}$ & $291.03^{\# \#}(279.28-298.55)$ \\
\hline MMP-7 (ng/ml) & $2.18^{\#}(2.13-2.23)$ & & & $3.83(3.75-3.89)^{*}$ & $4.16^{\# \#}(4.14-4.23)$ \\
\hline MMP-9 (ng/ml) & $97.2^{\#}(91.8-100.8)$ & & & $171.1(164.8-174.3)^{*}$ & $624.6^{\# \#}(594.4-650.0)$ \\
\hline TIMP-1 (ng/ml) & $82.3^{\#}(80.4-85.1)$ & & & $145.1(136.6-146.5)^{*}$ & $159.2^{\# \#}(152.0-163.3)$ \\
\hline TIMP-2 (ng/ml) & $41.88^{\#}(39.5-43.45)$ & & $3.05)$ & $152.75(147.35-157.55)^{*}$ & $171.95^{\# \#}(155.5-176.2)$ \\
\hline
\end{tabular}

Mann-Whitney U test: ${ }^{\#} p<0.0001$ APD, HD vs. control group, ${ }^{\# \#} p<0.001$ APD vs. after HD

Wilcoxon test: $* p<0.0001$ after HD vs. before HD

In all patients high sensitivity CRP, as a marker of inflammation (nephelometry by Dade Behring, Marburg, Germany), was also evaluated.

\section{Statistical analysis}

Results are expressed as median values and interquartile ranges. Differences between all groups and between the values before and after a single dialysis session were evaluated by using nonparametric tests (Kruskall-Wallis, Mann-Whitney U, Wilcoxon). The relations between parameters were assessed by Spearman's correlation coefficient and by linear regression analysis. The linear regression equations were calculated as $\mathrm{y}=\beta \mathrm{x}+\mathrm{a}$ (y is the dependent variable, $\beta$ is the regression coefficient, $\mathrm{x}$ is the independent variable, $a$ is the constant term). We presented only those equations where both regression coefficient and constant term were statistically significant. Statistical analysis was performed using the package Statistica ver. 9.0. A $p$ value $<0.05$ was considered significant.

\section{Results}

sFas, sFasL

sFas and sFasL median values were significantly higher in all dialyzed patients when compared to controls $(p<0.01)$ and the levels in the HD subgroup were higher than in the APD subjects (Figs. 1, 2). A single hemodialysis session decreased the concentrations of both parameters to the values below those seen in children on APD, yet they remained increased vs. controls.

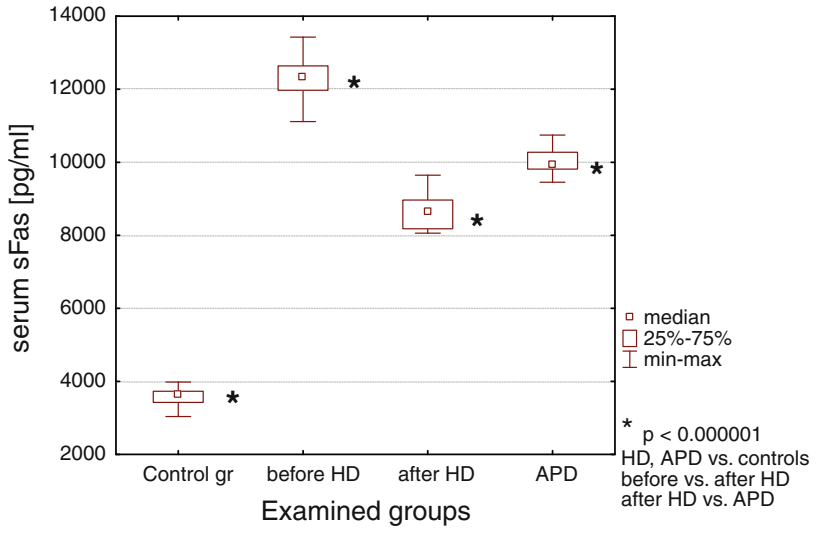

Fig. 1 Serum sFas concentrations in patients on HD before and after a single session, in children on APD and in controls

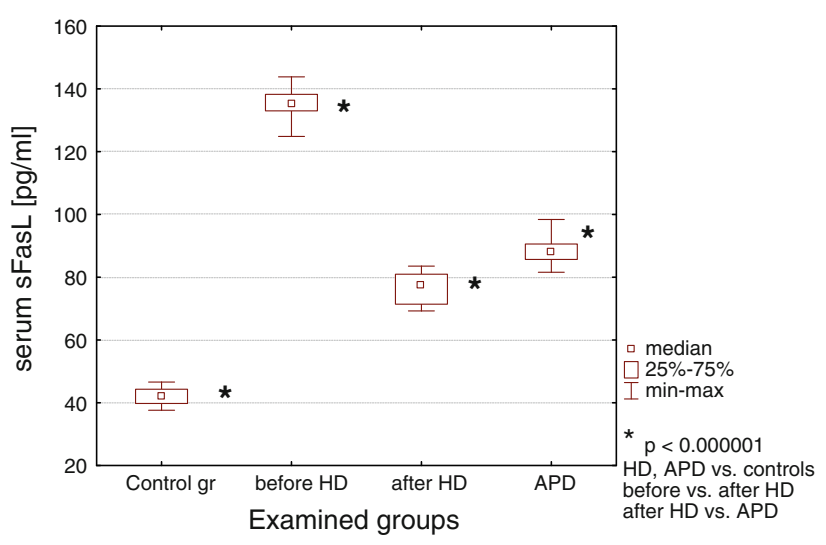

Fig. 2 Serum sFasL concentrations in patients on HD before and after a single session, in children on APD and in controls 
sFas/sFasL ratio

The values of the ratio were elevated in children on dialysis in comparison to the control group, being the highest in the hemodialyzed subjects (Fig. 3). The HD session diminished them, but without significant difference between post-hemodialysis and APD patients.

\section{MMPs and TIMPs}

The median values of MMP-2, MMP-7, MMP-9, TIMP-1 and TIMP-2 were increased in the population on dialysis when compared to the controls and the highest values were observed in patients on hemodialysis (Table 1). A single HD session diminished their concentrations to the levels lower than those observed in children on peritoneal dialysis (Table 1).

\section{hsCRP}

High sensitivity CRP levels did not show significant differences between dialyzed children and controls (Table 1), and a single HD session had no impact on its concentration.

\section{Linear regression analysis}

In all dialyzed patients, sFas, sFasL, MMPs and TIMPs correlated with each other (Table 2). Although all MMPs and TIMPs predicted the values of sFas and sFasL (Table 3), MMP-7 and TIMP-1 were the most accurate ones.

No associations between sFas/sFasL and hsCRP were observed.

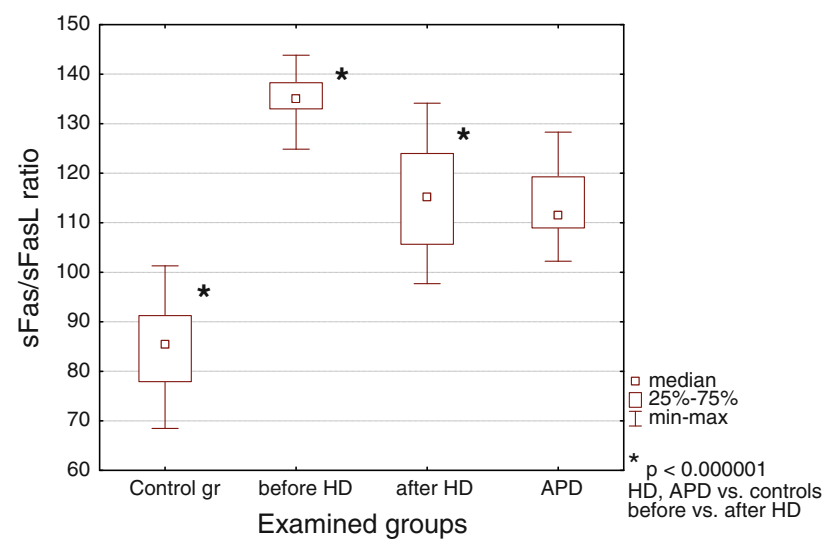

Fig. 3 The values of sFas/sFasL ratio in patients on HD before and after a single session, in children on APD and in controls

\section{Discussion}

Our study describes for the first time the disturbed sFas/ sFasL system, and the potential role of MMPs and TIMPs in its regulation, in children with end stage renal failure on dialysis.

sFas concentrations were increased in all patients on dialysis, which is concordant with previous observations in adults $[17,19,20]$. Such elevation may be a consequence of molecule accumulation due to decreasing GFR [19]. Perianayagam et al. have noticed that sFas levels in dialysed patients were higher than in pre-dialysis subjects, which may point at the dialysis per se as a procedure aggravating sFas release [19]. The accelerated synthesis of sFas, as a protective anti-apoptotic mechanism, cannot be neglected either [21]. sFas levels increase also with age [22]. Indeed, when we compared the concentrations of sFas in corresponding groups of children and adults [19], the latter were much higher. Additionally, sFas is a marker of atherosclerosis, endothelial dysfunction and coronary artery calcification-features characteristic for uremia [16, 23, 24].

However, the results concerning differences in sFas concentrations between various dialysis modalities are inconsistent. The probable source of discrepancies might be the difference between examined populations, concerning number of subjects and their age. However, the impact of methodology cannot be neglected either. Perianayagam et al. [19] reported on sFas levels being higher in patients on PD than in those on high-flux hemodialysis, whereas Dalboni et al. [17] found no difference between adults on continuous ambulatory peritoneal dialysis (CAPD) and subjects dialyzed on reused polysulfone membranes. None of the above mentioned conditions were similar to ours, because we examined children, performing APD and low-flux HD on first-use dialysers.

This is also the first report on the influence of a single hemodialysis session on sFas concentrations. The postdialysis drop in sFas levels may be of various origins. As a middle molecule $(\sim 50 \mathrm{kd})$, it can be only partly cleared during hemodialysis procedure. However, the adsorption on the membrane surface, as well as formation of sFassFasL complexes, also have to be taken into account. Although a single HD session was able to correct the sFas discrepancies to the levels below those seen in APD, this effect seems transient. It is probable, however, that the enlargement of total hemodialysis dose, e.g. by increasing its frequency, would ameliorate the patients' status by diminishing the overall apoptotic activity and avoiding sinusoidal changes in sFas concentrations between subsequent sessions. The high sFas levels in patients on chronic dialysis may also picture an attempt to protect vulnerable tissues against apoptosis, and in a long term 
Table 2 Correlations between examined parameters in all children on dialysis (APD + HD)

\begin{tabular}{llllll}
\hline Parameter & MMP-2 & MMP-7 & MMP-9 & TIMP-1 & TIMP-2 \\
\hline sFas & $R=0.23$ & $R=0.79$ & $R=0.68$ & $R=0.84$ & $R=0.69$ \\
& $p=0.14$ & $p=0.0000001$ & $p=0.000002$ & $p=0.0000001$ & $p=0.000001$ \\
sFasL & $R=0.38$ & $R=0.72$ & $R=0.62$ & $R=0.73$ & $R=0.79$ \\
& $p=0.02$ & $p=0.0000001$ & $p=0.00003$ & $p=0.0000001$ & $p=0.0000001$ \\
sFas/sFasL ratio & $R=-0.45$ & $R=-0.67$ & $R=-0.61$ & $R=-0.67$ & $R=-0.81$ \\
& $p=0.004$ & $p=0.000003$ & $p=0.00004$ & $p=0.000003$ & $p=0.0000001$ \\
\hline
\end{tabular}

$R$ Spearman's rank coefficient

Table 3 The linear regression analysis of assessed parameters in all children and young adults on dialysis (APD + HD)

\begin{tabular}{|c|c|c|c|c|c|}
\hline Dependent variable & Independent variable & $\begin{array}{l}\text { Regression } \\
\text { coefficient } \beta\end{array}$ & $\begin{array}{l}\text { Constant } \\
\text { term }\end{array}$ & $\begin{array}{l}\text { Coefficient of } \\
\text { determination } R^{2}\end{array}$ & $p$ \\
\hline \multirow[t]{6}{*}{ sFas (pg/ml) } & MMP-2 (ng/ml) & 13.03 & 7155.71 & 0.14 & 0.02 \\
\hline & MMP-7 (ng/ml) & 4051.91 & -6827.72 & 0.85 & 0.000001 \\
\hline & MMP-9 (ng/ml) & 11.87 & 2907.77 & 0.55 & 0.001 \\
\hline & TIMP-1 (ng/ml) & 8.31 & 8781.47 & 0.90 & 0.000001 \\
\hline & TIMP-2 (ng/ml) & 20.94 & 6799.97 & 0.66 & 0.00001 \\
\hline & sFasL (pg/ml) & 48.88 & 5762.81 & 0.85 & 0.000001 \\
\hline \multirow[t]{5}{*}{$\mathrm{sFasL}(\mathrm{pg} / \mathrm{ml})$} & MMP-2 (ng/ml) & 0.27 & 26.73 & 0.17 & 0.01 \\
\hline & MMP-7 (ng/ml) & 77.53 & -233.89 & 0.87 & 0.000001 \\
\hline & MMP-9 (ng/ml) & 0.23 & -51.28 & 0.59 & 0.0002 \\
\hline & TIMP-1 (ng/ml) & 0.16 & 64.52 & 0.94 & 0.000001 \\
\hline & TIMP-2 (ng/ml) & 0.44 & 19.77 & 0.80 & 0.00003 \\
\hline \multirow[t]{5}{*}{ sFas/sFasL ratio } & MMP-2 (ng/ml) & -0.14 & 146.37 & 0.15 & 0.02 \\
\hline & MMP-7 (ng/ml) & -35.50 & 261.28 & 0.63 & 0.000001 \\
\hline & MMP-9 (ng/ml) & -0.11 & 177.47 & 0.42 & 0.00007 \\
\hline & TIMP-1 (ng/ml) & -0.07 & 124.82 & 0.69 & 0.000001 \\
\hline & TIMP-2 (ng/ml) & -0.22 & 149.07 & 0.70 & 0.000001 \\
\hline
\end{tabular}

perspective-diminish the risk of vascular calcification [25]. However, although promising, this theory needs to be verified in the future investigation.

Studies evaluating sFasL in the population on chronic dialysis are even less numerous that those concerning sFas. Perianayagam et al. [19] observed no difference between the concentrations of sFasL in healthy controls, pre-dialysis subjects and those on dialysis, irrespective of the method used. Kirmizis et al. [26] found no improvement in apoptosis markers after a 6-month course of hemodialysis on vitamin E-coated membranes vs. other membranes used earlier, but they did not compare those values with the control group of healthy subjects. In our population, sFasL behaved similarly to $\mathrm{sFas}$ - there was a significant increase in all dialyzed children, with preponderance in those on hemodialysis. The sFasL elevation might result from the combination of mFasL overexpression on inflammatory cells, characteristic for increased apoptosis, and overactivity of MMPs and TIMPs, additionally triggering the sFasL shedding [27]. The accumulation due to renal failure is also possible, although the inverse correlation, existing between GFR and sFas, has not been confirmed in the case of sFasL. A single HD session diminished the sFasL concentrations to the values lower than those in APD patients. That decrease, even more evident than in the case of sFas, may be attributed to the fact that sFasL is a molecule smaller that sFas ( $\sim 30 \mathrm{vs.} 50 \mathrm{kd})$ and its clearance is more effective. The adsorption on the membrane surface and formation of sFas-sFasL complexes, should also be taken into account as alternative explanations. Last, but not least, the long-term effect of the transient intradialytic leukopenia cannot be neglected either.

However, the unresolved problem is whether sFasL, due to its decreased apoptotic potential vs. mFasL, should be regarded as an anti- or pro-apoptotic factor. Indeed, when the increased overall activity of mFasL on immunocompetent cells in uremia is considered, the weaker proapoptotic impact of sFasL vs. mFasL cannot compensate the overexpression of the latter and, therefore, cannot be interpreted solely as an anti-apoptotic action. Likewise, the 
fact that $\mathrm{sFas}$ and $\mathrm{sFasL}$ antagonize each other by stoichiometric binding 1:1, speaks in favor of their ambiguous activities. Moreover, recent investigation in knockout mice has suggested that high levels of sFasL may trigger autoimmune reactions and promote tumorigenesis [28].

Therefore, the best way to assess the overall apoptotic activity would rather be to evaluate the proportion between the concentrations of sFas and sFasL. The values of sFas/ sFasL ratio followed the same pattern as both parameters analyzed separately, with one exception. The difference between post-hemodialysis and APD subjects has vanished, suggesting that the overall apoptotic ability of patients on peritoneal dialysis is comparable to that in patients after a HD session. Consequently, subjects on hemodialysis are prone to fluctuations, whereas children on peritoneal dialysis seem fairly stable, which is of paramount importance, as this modality is a method of choice in pediatric population with end stage renal disease.

The subject of sFas/sFasL relation to MMPs and TIMPs has never been described in patients with chronic kidney disease. We have previously reported on increased concentrations of MMP-7 and TIMP-1 in children on chronic dialysis, with their values being higher in hemodialyzed patients than in those on peritoneal dialysis [29]. Our currently extended panel has also covered gelatinases (MMP-2, MMP-9) and TIMP-2, showing the similar pattern of discrepancy between children on APD and HD. Like in the case of sFasL, all parameters decreased after a HD session to the levels below those seen in APD children, probably due to both adsorption on the dialysis membrane and formation of MMP-TIMP complexes. This concordance could also result from the potential regulatory role of MMPs and TIMPs in shedding sFasL from its membranebound form mFasL. Moreover, all MMPs and TIMPs could predict $\mathrm{sFasL}$ concentrations in the dialyzed population. Among these, MMP-7 and TIMP-1 turned out to be the best predictors of sFaL. While MMP-7 occupies an established position of an enzyme critical in sFasL formation [10-12], the influence of TIMP-1 on the process is a new finding. Nevertheless, the fact that TIMP-1 is a MMP-9 specific inhibitor, and the suppression of the latter promotes apoptosis, could render a probable explanation for the observed correlation [30]. The discrepancies in accuracy of prediction between various MMPs and TIMPs may result from alternative cleavage sites, thus creating forms with different activity and individual apoptotic potential [9].

Another intriguing question is whether MMPs and TIMPs can also take part in the regulation of sFas shedding, thus influencing apoptosis, inflammation and endothelial function. So far, the only known way of sFas synthesis is that by the alternatively spliced mRNA, so the role of MMPs and TIMPs in mFasL cleavage seems questionable [31]. However, sFasL correlations with proteases may merely suggest that those enzymes could be considered markers of inflammation and endothelial dysfunction. Therefore, the real nature of relations between sFas, MMPs and TIMPs requires further investigation.

\section{Summary}

Children and young adults on chronic dialysis present with $\mathrm{sFas} / \mathrm{sFasL}$ system dysfunction and increased apoptotic activity, less pronounced in patients on peritoneal dialysis. The correlations between sFas/sFasL, MMPs and TIMPs suggest that those enzymes may influence the programmed cell death by stimulating both protective (sFas) and destructive (sFasL) mechanisms responsible for apoptosis in that group of patients.

Open Access This article is distributed under the terms of the Creative Commons Attribution Noncommercial License which permits any noncommercial use, distribution, and reproduction in any medium, provided the original author(s) and source are credited.

\section{References}

1. Andreoli MCC, Dalboni MA, Watanabe R, Manfredi SR, Canziani MEF, Kallas EG, Sesso RC, Draibe SA, Balakrishnan VS, Jaber BL, Liangos O, Cendoroglo M (2007) Impact of dialyzer membrane on apoptosis and function of polymorphonuclear cells and cytokine synthesis by peripheral blood mononuclear cells in hemodialysis patients. Artif Org 31:887-910

2. Soriano S, Martin-Malo A, Carracedo J, Ramirez R, Rodriguez M, Aljama P (2005) Lymphocyte apoptosis: role of uremia and permeability of dialysis membrane. Nephron Clin Pract 100: c71-c77

3. Nagata S, Goldstein P (1995) The Fas death factor. Science 267:1449-1456

4. Yang Z, Gagarin D, Ramezani A, Hawley RG, McCaffrey TA (2007) Resistance to Fas-induced apoptosis in cells from human atherosclerotic lesions: elevated $\mathrm{Bcl}-\mathrm{X}_{\mathrm{L}}$ inhibits apoptosis and caspase activation. J Vasc Res 44:483-494

5. McCaffrey TA, Du B, Fu Ch, Bray PJ, Sanborn TA, Deutsch E, Tarazona N, Shaknovitch A, Newman G, Patterson C, Bush HL Jr (1999) The expression of TGF- $\beta$ receptors in human atherosclerosis: evidence for acquired resistance to apoptosis due to receptor imbalance. J Mol Cell Cardiol 31:1627-1642

6. Clarke MC, Figg N, Maguire JJ, Davenport AP, Goddard M, Littlewood TD, Bennett MR (2006) Apoptosis of vascular smooth muscle cells induces features of plaque vulnerability in atherosclerosis. Nat Med 12:1075-1080

7. Matter ChM, Chadjichristos ChE, Meier P, von Lukowicz T, Lohmann Ch, Schuler PK, Zhang D, Odermatt B, Hofmann E, Brunner T, Kwak BR, Luescher T (2006) Role for endogenous Fas (CD95/Apo-1) ligand in balloon-induced apoptosis, inflammation, and neointima formation. Circulation 113:1879-1887

8. Kayagaki N, Kawasaki A, Ebata T, Ohmoto H, Ikeda S, Inoue S, Yoshino K, Okumura K, Yagita H (1995) Metalloproteinasemediated release of human Fas ligand. J Exp Med 182:1777-1783 
9. Vargo-Gogola T, Crawford HC, Fingleton B, Matrisian LM (2002) Identification of novel matrix metalloproteinase-7 (matrilysin) cleavage sites in murine and human Fas ligand. Arch Biochem Biophys 408:155-161

10. Poulaki V, Mitsiades CS, Mitsiades N (2001) The role of Fas and FasL as mediators of anticancer chemotherapy. Drug Resist Updates 4:233-242

11. Liu H, Huang J, Wu B, Zhou Y, Zhu J, Zhang T (2008) Matrilysin inhibits proliferation and modulates sensitivity of lung cancer cells to FasL-mediated apoptosis. Med Oncol 25:419-430

12. Tsukinoki T, Sugiyama H, Sunami R, Kobayashi M, Onoda T, Maeshima Y, Yamasaki Y, Makino H (2004) Mesangial cell Fas ligand: upregulation in human lupus nephritis and NK- $\kappa \mathrm{B}$-mediated expression in cultured human mesangial cells. Clin Exp Nephrol 8:196-205

13. Schneider P, Holler N, Bodmer JL, Hahne M, Frei K, Fontana A, Tschopp J (1998) Conversion of membrane-bound Fas (CD95) ligand to its soluble form is associated with downregulation of its proapoptotic activity and loss of liver toxicity. J Exp Med 187:1205-1213

14. Tanaka M, Itai T, Adachi M, Nagata S (1998) Downregulation of Fas ligand by shedding. Nat Med 4:31-36

15. El-Agroudy AE, El-Baz A (2010) Soluble Fas: a useful marker of inflammation and cardiovascular diseases in uremic patients. Clin Exp Nephrol 14:152-157

16. Dalboni MA, Sardenberg C, Andreoli MC, Watanabe R, Canziani ME, dos Santos BFC, Liangos OL, Jaber BL, Draibe S, Cendoroglo M (2003) Soluble Fas: a novel marker of inflammation in uremia. Artif Org 27:687-691

17. Wang T, Dong Ch, Stevenson SC, Herderick EE, Marshall-Neff J, Vasudevan SS, Moldovan NI, Michler RE, Movva NR, Goldschmidt-Clermont PJ (2002) Overexpression of soluble Fas attenuates transplant arteriosclerosis in rat aortic allografts. Circulation 106:1536-1542

18. National High Blood Pressure Education Program Working Group on High Blood Pressure in Children and Adolescents (2004) The fourth report on the diagnosis, evaluation, and treatment of high blood pressure in children and adolescents. Pediatrics 114:555-576

19. Perianayagam MC, Murray SL, Balakrishnan VS, Guo D, King AJ, Pereira BJG, Jaber BL (2000) Serum soluble Fas (CD95) and Fas ligand profiles in chronic kidney failure. J Lab Clin Med 136:320-327

20. Goes MA, Dalboni MA, Manfredi SR, Cendoroglo MS, Batista MC, Canziani ME, Balakrishnan VS, Pereira BJ, Draibe SA, Cendoroglo M (2010) Serum-soluble Fas and serum levels of erythropoietin in chronic kidney disease. Clin Nephrol 73:7-13
21. Dalboni MA, Cenedeze MA, Manfredi SR, Cruz Andreoli MC, Pavao Dos Santos O, Canziani ME, Boim MA, Goes MA, Draibe SA, Balakrishnan V, Cendoroglo M (2008) High serum levels of soluble Fas (sFas) in CKD patients: effects of renal clearance, reabsorption and synthesis. Int J Artif Organs 31:405-410

22. Seishima M, Takemura M, Saito K, Sano H, Minatoguchi S, Fujiwara H, Hachiya T, Noma A (1996) Highly sensitive ELISA for soluble Fas in serum: increased soluble Fas in the elderly. Clin Chem 42:1911-1914

23. Masse M, Hebert MJ, Troyanov S, Vigneault N, Sirois I, Madore F (2002) Soluble Fas is a marker of peripheral arterial occlusive disease in haemodialysis patients. Nephrol Dial Transplant 17:485-491

24. Ammirati AL, Dalboni MA, Cendoroglo M, Draibe SA, Canziani MEF (2006) Coronary artery calcification, systemic inflammation markers an mineral metabolism in a peritoneal dialysis population. Nephron Clin Pract 104:c33-c40

25. Proudfoot D, Skepper JN, Hegyi L, Bennett MR, Shanahan CM, Weissberg PL (2000) Apoptosis regulates human vascular calcification in vitro. Evidence for initiation of vascular calcification by apoptotic bodies. Circ Res 87:1055-1062

26. Kirmizis D, Papagianni A, Belechri AM, Memmos D (2010) Effects of vitamin E-coated membrane dialyser on markers of oxidative stress and inflammation in patients on chronic haemodialysis. Nephrol Dial Transplant. doi:10.1093/ndt/gfq715

27. Jaber BL, Perianayagam MC, Balakrishnan VS, King AJ, Pereira BJ (2001) Mechanisms of neutrophil apoptosis in uremia and relevance of the Fas (APO-1, CD95)/Fas ligand system. J Leukoc Biol 69:1006-1012

28. O'Reilly LA, Tai L, Lee L, Kruse EA, Grabow S, Fairlie D, Haynes NM, Tarlinton DM, Zhang JG, Belz GT, Smyth MJ, Bouillet P, Robb L, Strasser A (2009) Membrane-bound but not secreted Fas ligand is essential for Fas-induced apoptosis and prevention of autoimmunity and cancer. Nature 461:659-663

29. Musiał K, Zwolińska D (2011) Neutrophil gelatinase-associated lipocalin (NGAL) and matrix metalloproteinases as novel stress markers in children and young adults on chronic dialysis. Cell Stress Chaperones 16:163-171

30. Nalla AK, Gorantla B, Gondi CS, Lakka SS, Rao JS (2010) Targeting MMP-9, uPAR, and cathepsin B inhibits invasion, migration and activates apoptosis in prostate cancer cells. Cancer Gene Ther 17:599-613

31. Cheng J, Zhou T, Liu C, Shapiro JP, Brauer MJ, Kiefer MC, Barr PJ, Mountz JD (1994) Protection from Fas-mediated apoptosis by a soluble form of the Fas molecule. Science 263:1759-1762 